\title{
KINEMATICAL SIMULATION METHODS FOR STEWART PLATFORM IN MEDICAL EQUIPMENTS
}

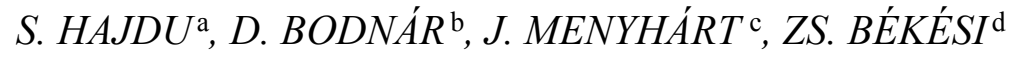 \\ Department of Mechanical Engineering, University of Debrecen, 4028 Debrecen, Ótemető u. 2-4, Hungary \\ aE-mail: hajdusandor@eng.unideb.hu \\ bE-mail: bodnar.david2@gmail.com \\ cE-mail: jozsef.menyhart@eng.unideb.hu \\ dE-mail: zsolt.bekesi@eng.unideb.hu
}

\begin{abstract}
The efficiency and accuracy of the modern positioning systems are crucial points in their design. The designers and engineers are highly motivated to find new methods and solutions to the accuracy problem with the biggest mobility and safety. Considerable numbers of solutions are on the market but only some of them can be used in medical industry. The authors will propose some kinematical simulation methods using MATLAB ${ }^{\circledR}$ software. The purpose of these methods is to use Stewart Platform with a better accuracy. The results show that it is possible to simulate Stewart Platform in MATLAB ${ }^{\circledR}$ and it can be used in medical industry.
\end{abstract}

Keywords: Stewart platform, hexapod, kinematical simulation, parallel manipulators, six degree freedom manipulators

\section{Introduction}

Nowadays we can find different types of positioning systems and holders in the industrial life. We have to accept the fact of accuracy is very important. The machines and equipments work with different accuracy, it means that we can find disparate solutions in the different industrial areas. These kinds of facts get a higher priority in medical industry especially in cancer operation systems like gamma knives. The engineers would like to simulate the patient holding equipments with a higher efficiency. In the modern research and development centres the engineers use large amount of mathematical software packages to find or specify the ideal parameters for the patient holding equipments. Thanks to these programs the calculations and simulations are much easier than ever before.

The engineers try to use different types of patient position equipments during their research. The aim of this article is a patient positioning system which is based on a Stewart Platform system.

The authors had leaned on a simulation executed over a Stewart Platform of a patient positioning system at University of Debrecen. The article is based on studies and experiences, from previous works from material handling science and MATLAB ${ }^{\circledR}$ simulations
[1-4]. The motivation of the authors is to find an ideal method for Stewart Platform simulation design which can give a better accuracy for the patient holding system and it can give a better design solution for a high accuracy Stewart Platforms.

During the simulation process, the authors work with MATLAB ${ }^{\circledR}$ on inverse and direct kinematic calculations. The study presents a method of Stewart Platform simulation in MATLAB ${ }^{\circledR}$ environment.

\section{Positioning solutions - Stewart Platform}

The first Stewart Platform was developed and designed by the Performance and Stressing Department of the New Tyre Design and Development Division, at Dunlop Rubber Corporation in Birmingham, England. This Stewart Platform was installed in 1954 [7-10]. This platform was used for flight simulators, machine tools and universal milling machines in 1965.

The Stewart Platform is sometimes also referred as Gough - Stewart Platform or Hexapod. It always depends on the used references [7-10]. Basically the Stewart Platform is a type of parallel robot which has six prismatic actuators.

The platform commonly works with hydraulic jacks or electric actuators. These are attached in pairs

This is an open-access article distributed under the terms of the Creative Commons Attribution-NonCommercial 4.0 International License (https://creativecommons.org/licenses/by-nc/4.0/), which permits unrestricted use, distribution, and reproduction in any medium for non-commercial purposes, provided the original author and source are credited, a link to the CC License is provided, and changes - if any - are indicated. 
to three positions on the base of the platform. The whole machine can move in six degrees of freedom in which it is possible for a freely suspended body to move. These are the three linear movements, the axes are: $x, y, z$. It means lateral, longitudinal and vertical and three rotations pitch, roll and yaw.

Until the near past this kind of equipments needed a high power and expensive computers. Nowadays the engineer can use relatively low cost computers. It means that the machine tool application can now be controlled on a low cost.

The Stewart Platform can be used in a wide area of industrial fields, e.g. flight simulations, material handling and logistic equipments, low impact docking systems, etc. [7-10]. Nowadays the hexapod systems are getting a higher priority in medical industry, especially in medical machines, like operating systems. A RSPR3 manipulator is shown with an arthoscopic camera [11].

The previous example shows an equipment which is useful in health diagnostics. But in a modern robotic field the hexapods are working in different surgery equipments $[12,13]$. The examples below show machines where the patient is in a fix position. The scope of the study is to simulate and analyse hexapods when the platform tries to move the patient into the right position during the surgery. This platform has an important task. It has to compensate the small patient displacements, like breath and sneezing. The accuracy of these kinds of machines is crucial.

The next section presents kinematic calculations and MATLAB ${ }^{\circledR}$ simulations of Stewart platforms.

\section{Inverse kinematic calculations for two types of Stewart Platforms}

\subsection{Inverse kinematic of the 6-6 Stewart Platform}

Figure 1 shows a general Stewart platform constructed from a hexagon base and a hexagon platform. The two platforms are connected with six linear actuators, also called legs. The base has its own coordinate system $X Y Z$, with a centre point $\mathrm{O}$. The platform also has

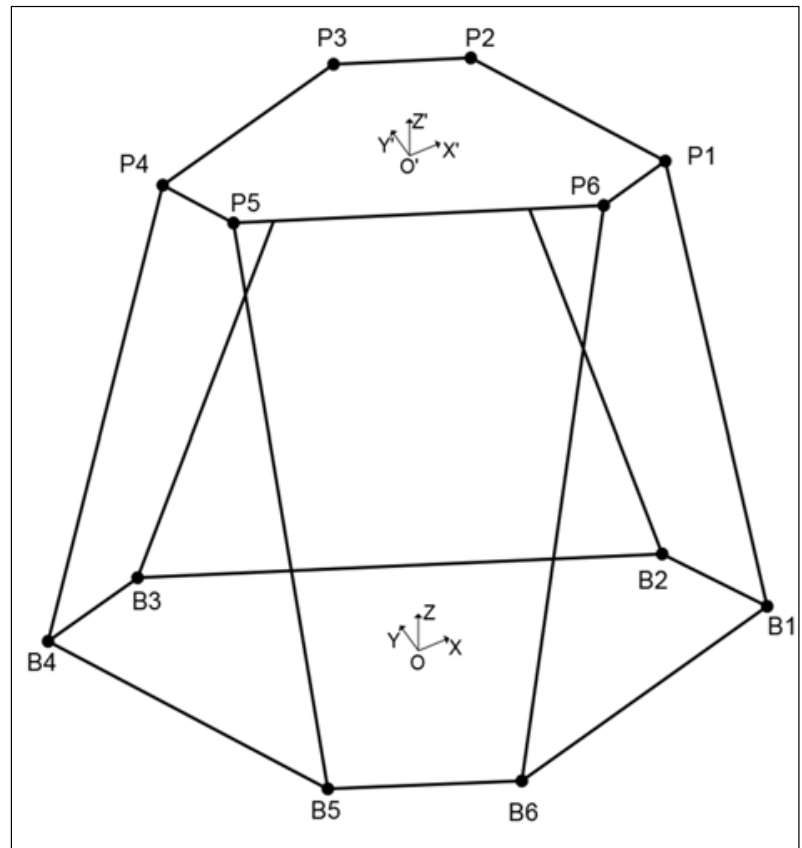

Fig. 1. General 6-6 Stewart Platform

its own coordinate system with $X^{\prime}-Y^{\prime}-Z^{\prime}$ axes, and a centre point $\mathrm{O}^{\prime}$.

We wanted to develop a flexible solution, that can calculate the base and the platform anchor point positions based on minimal input data. We decided that if we divide the platform into three segments by $120^{\circ}$ we can then define a $\sigma$ value for calculating the vectors $\bar{b}_{i}$ and $\bar{p}_{i}$. All the anchor points can be defined by the radius, and that value.

The position that we want our platform to be can be expressed by the $X Y Z$ coordinates and a set of Euler angles in the following sequence:

1. rotate an angle $\psi$ (yaw) around the $z$ axis,

2. rotate an angle $\theta$ (pitch) around the $y$ axis,

3 . rotate an angle $\varphi$ (roll) around the $x$ axis.

The next element we need is a rotation matrix to convert the values from the platform coordinate system into the default base coordinate system. That we can write in the following form:

$$
R=\left[\begin{array}{ccc}
\cos \psi \cos \theta & -\sin \psi \cos \varphi+\cos \psi \sin \theta \sin \varphi & \sin \psi \sin \varphi+\cos \psi \sin \theta \cos \varphi \\
\sin \psi \cos \theta & \cos \psi \cos \varphi+\sin \psi \sin \theta \sin \varphi & -\cos \psi \sin \varphi+\sin \psi \sin \theta \cos \varphi \\
-\sin \theta & \cos \theta \cos \varphi
\end{array}\right]
$$

The length of the legs needs to be calculated by the following method. First, we calculate the position of the leg connection points $B_{i}$ in the base, with respect to the base coordinate system, and we also calculate the leg connection points $P_{i}$ for the platform, in $X^{\prime} Y^{\prime} Z^{\prime}$ coordinates. After that we can calculate the coordinates of the $q_{i}$ anchor point by the following formula:

$$
\bar{q}_{i}=\bar{t}+R * \bar{p}_{i}
$$

where $\bar{t}$ expresses the relation between the base origin and the platform origin, $\bar{p}_{i}$ defines the coordinates of the $P_{i}$ point, and needs to be multiplied with the rotation matrix to convert it to the base coordinate system. 


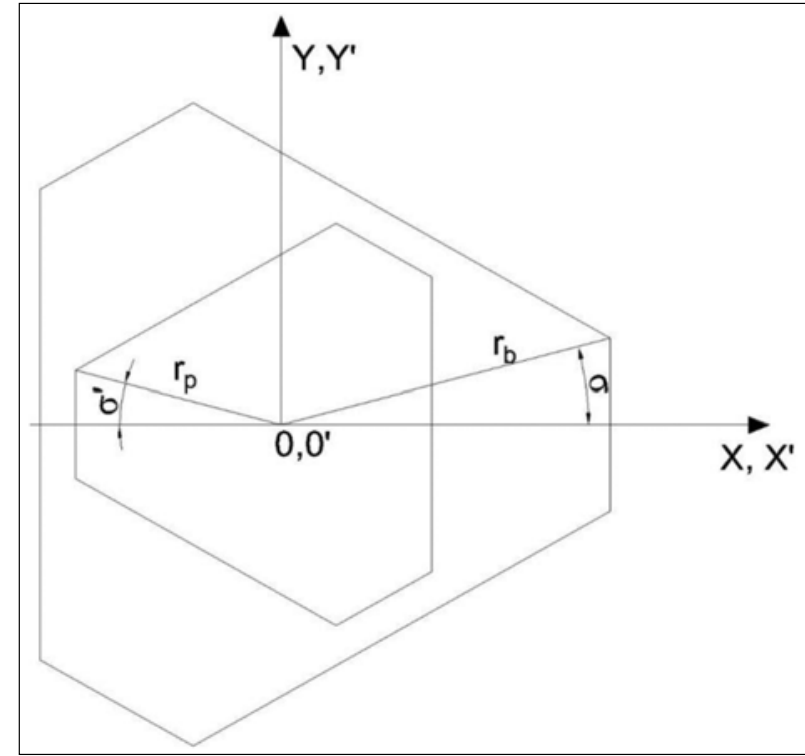

Fig. 2. Construction of the base and the platform

The length of the $i$ th leg is given by:

$$
\left|\bar{l}_{i}\right|=\left|\bar{t}+R * \bar{p}_{i}-\bar{b}_{i}\right|
$$

where $\bar{b}_{i}$ is the vector that defines the coordinates of the $B_{i}$ point. That equation expressed for legs $1-6$ give the required leg lengths to achieve the desired position and attitude of the platform.

\subsection{Inverse kinematic of the rotation type Stewart Platform}

The rotation type Stewart Platform has fixed leg lengths. To achieve the desired positions it rotates the lower leg anchor points around a circular path, similar to Mikrolar's Rotopod.

We can solve the inverse kinematics of the rotation type Stewart Platform based on the calculations required for the generic type Stewart Platform. The upper leg anchor point coordinates $\bar{q}_{l}$ can be calculated with Eq. (2), but the problem is that we need to calculate the exact position of the lower anchor point defined by the fixed leg length. For that we came up with the following solution.

The circular path where the lower anchor points can be positioned is a circle located in the $X Y$ plane of the base coordinate system. It can be expressed by the equation:

$$
x^{2}+y^{2}=r_{b}^{2}
$$

We also know the coordinates for all six anchor points. If we place a sphere originated from the point and with the radius of the fixed leg length we get the following equation of a sphere:

$$
\left(x-\bar{q}_{l}(x)\right)^{2}+\left(y-\bar{q}_{l}(y)\right)^{2}+\left(z-\bar{q}_{l}(z)\right)^{2}=\left|\bar{l}_{\text {fix }}\right|^{2} .
$$

If we make these two equations equal to zero we get a nonlinear equation system for each leg. The solutions for these equation systems is only true where our circle and our sphere intersect each other. And these intersection points are where the lower leg anchor points need to achieve the desired platform attitude and rotation. We can also eliminate the $z$ value, since it will always be 0 in the $X Y$ plane of the base. The final nonlinear equation system:

$$
\bar{F}(\bar{x}, \bar{y})=\left[\begin{array}{c}
x^{2}+y^{2}=r_{b}^{2} \\
\left(x-\bar{q}_{i}(x)\right)^{2}+\left(y-\bar{q}_{i}(y)\right)^{2}+\left(z-\bar{q}_{i}(z)\right)^{2}=\left|\bar{l}_{\text {fix }}\right|^{2}
\end{array}\right]=0 .
$$

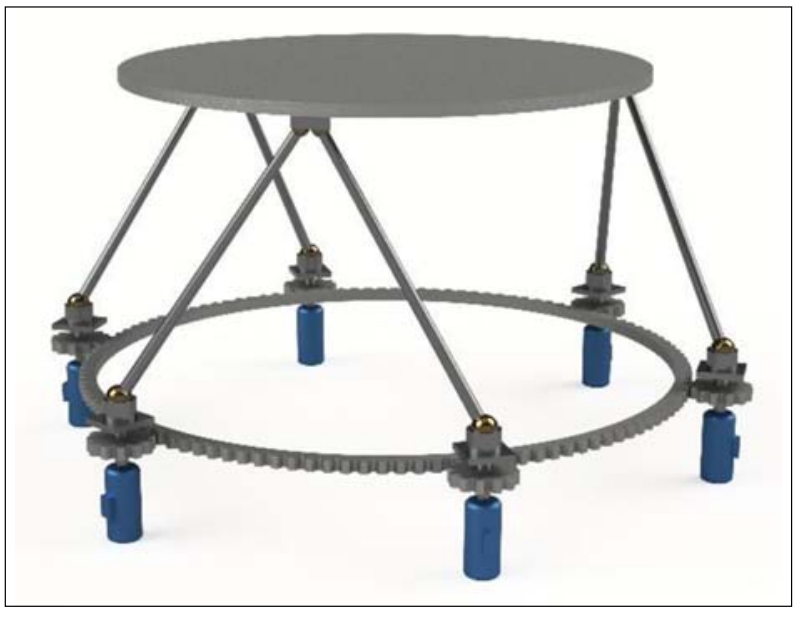

Fig. 3. Rotation type hexapod
The last problem that we need to solve is that there are always two intersection points, except for the boundary position. For that we use the MATLAB ${ }^{\circledR}$ function fsolve $\mathrm{x} 0$. We choose $\mathrm{x} 0$ to be the initial $b_{i}$ position of the leg, that way we arrive to only one solution, the one that is closer to the initial starting point.

\subsection{Forward kinematics of the Stewart Platform}

The forward kinematics solution for the Stewart Platform is very complex, because of the 6 unknowns representing the position and attitude of the platform. For that reason, it is very resource heavy to calculate it in real time. We plan to solve this problem using an FPGA controller, but until then we can implement an 
iterative method also used in the ACS SPiiPlus Controller. It works like this:

1. Estimate that the actual measured $(x, y, z, \psi, \theta, \varphi)$ position is the commanded position. We can call it $E$.

2. Use the inverse kinematics equations to find the corresponding leg lenghts $\left(l_{1}, \ldots, l_{6}\right)$.

3. Compare these values to the actual leg lenghts (measured by the encoders attached to the motors) to get a vector of errors $\bar{l}_{e}$. If $\bar{l}_{e}$ is less than the error threshold, then we are done. If not, continue.

4. Calculate the Jacobian matrix $J$ and then invert to get $J^{-1}$.

5. The new estimation $\left(E_{1}\right)$ is obtained by taking the original estimation and adding a factor $\left(E_{1}=E-J^{-1} * \bar{l}_{e}\right)$.

6. Go back to part 2 using the new estimation.

\subsection{Examples}

Example 1. Calculating the home position of the general type Stewart Platform

We want to construct our platform with the following properties:

$$
\begin{array}{ll}
r_{b}=100[\mathrm{~mm}], & \sigma=15^{\circ}, \\
r_{p} & =70[\mathrm{~mm}], \\
h & \sigma^{\prime}=15^{\circ}, \\
h &
\end{array}
$$

From that our MATLAB ${ }^{\circledR}$ script first calculates the vectors $\bar{b}_{l}$ and $\bar{p}_{l}$ (Table 1 ).
Next we enter the position we want to achieve, for this example it is: $\operatorname{Pos}(x, y, z, \psi, \theta, \varphi)=(0,0,0,0,0,0)$. The script returns the required leg lengths (Table 2 ).

Table 2. The required leg lengths

\begin{tabular}{lc}
\hline & $\bar{l}_{l}$ \\
\hline 1 & 83,6699 \\
2 & 83,6699 \\
3 & 83,6699 \\
4 & 83,6699 \\
5 & 83,6699 \\
6 & 83,6699 \\
\hline
\end{tabular}

Example 2. General type Stewart platform

We will leave the platform properties the same, but our new demanded position is: $\operatorname{Pos}(x, y, z, \psi, \theta, \varphi)$ $=\left(10,20,-10,5^{\circ},-15^{\circ},-2^{\circ}\right)$. The script returns the required leg lengths (Table 3 ).

Table 3. Required leg lengths

\begin{tabular}{lc}
\hline & $\left|\bar{l}_{l}\right|$ \\
\hline 1 & 77,8446 \\
2 & 92,0611 \\
3 & 75,2491 \\
4 & 55,0838 \\
5 & 78,9186 \\
6 & 99,2421 \\
\hline
\end{tabular}

Table 1. The vectors $\bar{b}_{l}$ and $\bar{p}_{l}$

\begin{tabular}{ccc}
\hline & $\bar{b}_{l}$ & $\bar{p}_{l}$ \\
\hline 1 & {$[96,5926 ;-25,8819 ; 0]$} & {$[49,4975 ;-49,4975 ; 0]$} \\
2 & {$[96,5926 ; 25,8819 ; 0]$} & {$[49,4975 ; 49,4975 ; 0]$} \\
3 & {$[-25,8819 ; 96,5926 ; 0]$} & {$[18,1173 ; 67,6148 ; 0]$} \\
4 & {$[-70,7107 ; 70,7107 ; 0]$} & {$[-67,6148 ; 18,1173 ; 0]$} \\
5 & {$[-70,7107 ;-70,7107 ; 0]$} & {$[-67,6148 ;-18,1173 ; 0]$} \\
6 & {$[-25,8819 ;-96,5926 ; 0]$} & {$[18,1173 ;-67,6148 ; 0]$} \\
\hline
\end{tabular}

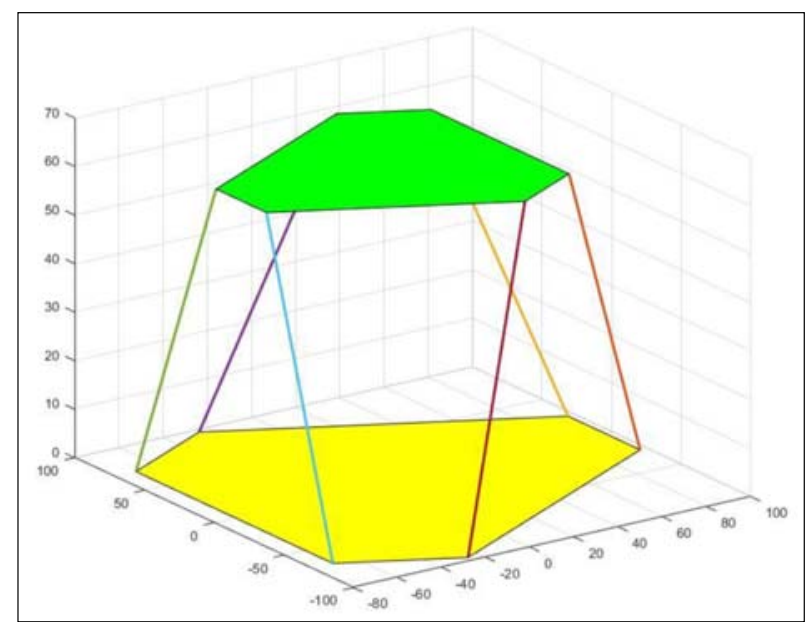

Fig. 4. Plot of Example 1

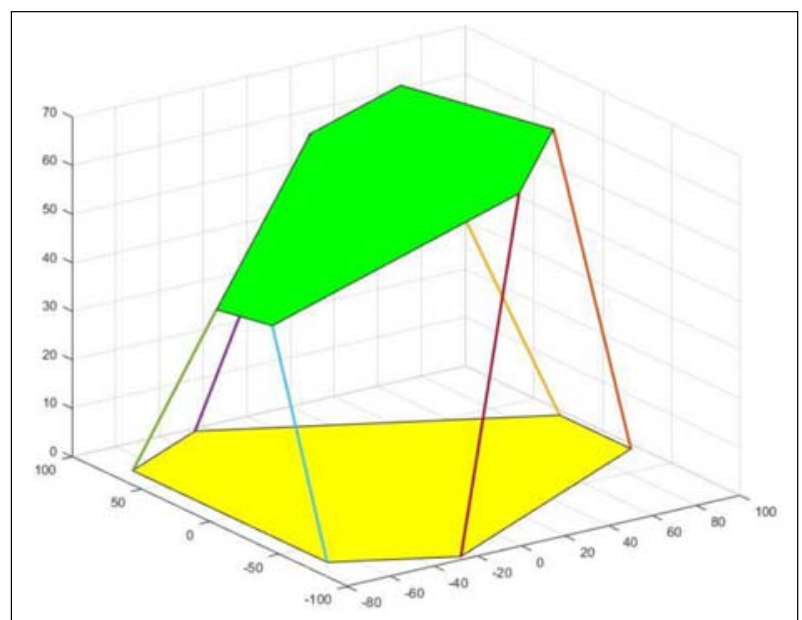

Fig. 5. Plot of Example 2 
Example 3. Rotation type Stewart Platform in home position

We will leave the platform properties the same, the only difference is that we use the $B_{i}$ points as the initial positions for legs along the circular path. We calculate the virtual leg lengths using Eq. (3). We use that data to calculate the new $B_{i}$ coordinates (Table 4).

Table 4. New coordinates $B_{i}$

\begin{tabular}{cc}
\hline & $B_{i}$ \\
\hline 1 & {$[96,6818 ;-25,5467 ; 0]$} \\
2 & {$[96,6818 ; 25,5467 ; 0]$} \\
3 & {$[-26,2168 ; 96,5022 ; 0]$} \\
4 & {$[-70,4650 ; 70,9555 ; 0]$} \\
5 & {$[-70,4650 ;-70,9555 ; 0]$} \\
6 & {$[-26,2168 ;-96,5022 ; 0]$} \\
\hline
\end{tabular}

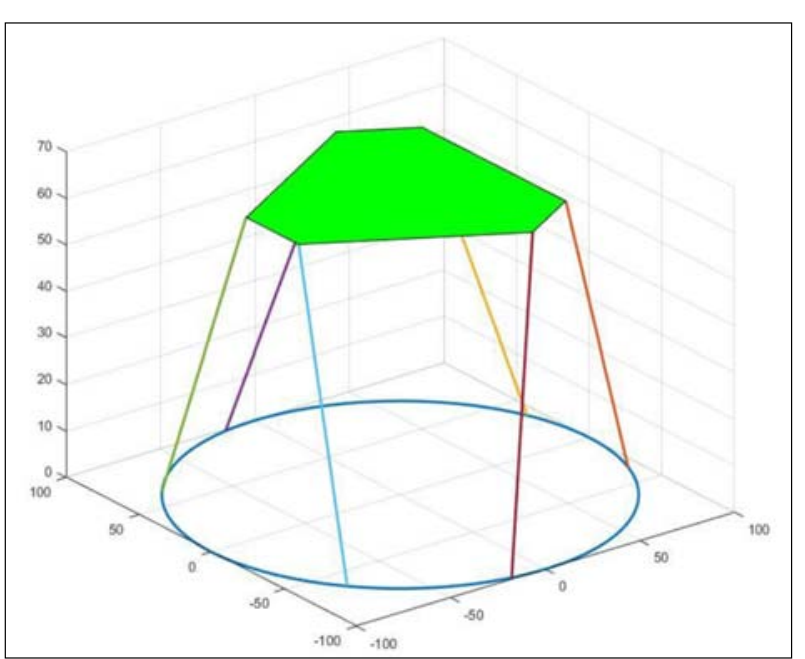

Fig. 6. Plot of Example 3

\section{Example 4. Rotation type}

We will leave the platform properties the same, but our new demanded position is: $\operatorname{Pos}(x, y, z, \psi, \theta, \varphi)=$ $\left(10,20,-5,0^{\circ},-15^{\circ}, 10^{\circ}\right)$. We calculate the virtual leg lengths with Eq. (3). We use that data to calculate the new $B_{i}$ coordinates (Table 5).

Table 5. New $B_{i}$ coordinates

\begin{tabular}{cc}
\hline & $B_{i}$ \\
\hline 1 & {$[99,9445 ;-3,3308 ; 0]$} \\
2 & {$[62,7641 ; 77,6225 ; 0]$} \\
3 & {$[3,4585 ; 99,9402 ; 0]$} \\
4 & {$[-26,3184 ; 96,4746 ; 0]$} \\
5 & {$[-77,1279 ;-63,6497 ; 0]$} \\
6 & {$[-3,9070 ;-99,9236 ; 0]$} \\
\hline
\end{tabular}

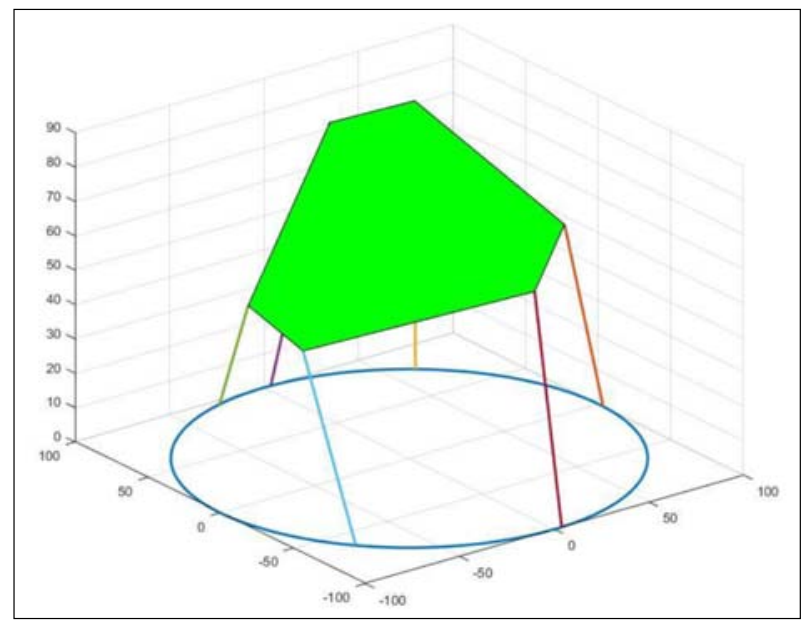

Fig. 7. Plot of Example 4

\section{Conclusion}

The authors discussed the results of the simulation. The conventional method and simulation based on inverse kinematic methods and formulas. The authors checked the main general properties and parameters of a Stewart Platform system and analyzed the kinematic properties with MATLAB ${ }^{\circledR}$ software. It can be concluded that using the Stewart platform is very promising, and plan to further develop the simulation using MATLAB $^{\circledR}$.

\section{Acknowledgements}

The work/publication is supported by the EFOP-3.6.116-2016-00022 project. The project is co-financed by the European Union and the European Social Fund.

\section{References}

[1] Szabolcsi R., Menyhart J. (2017), Battery voltage limit analysis with support vector machine and fuzzy logic. Advances in Military Technology, 12(1), 21-32.

[2] Pokorádi L., Menyhárt J. (2016), Electric vehicles' battery parameter tolerances analysis by Fuzzy Logic, Proceedings of the11th IEEE International Symposium on Applied Computational Intelligence and Informatics SACI 2016. 412 p. Conference venue, time: Timisoara, Romania, 2016.05.12-2016.05.14. Budapest: IEEE, 2016. pp. 361-364.

[3] Hajdu S., Gáspár P. (2014), From modeling to robust control design of single-mast stacker cranes. Acta Polytechn. Hung, 11(10), 135-149.

[4] Hajdu S., Gáspár P. (2016), Reducing the mast vibration of single-mast stacker cranes by gain-scheduled control. Int. J. Appl. Math. Comp. Science, 26(4), 791-802.

[5] Houdek P. J. (1997), Design and Implementation Issues for Stewart Platform Configuration Machine Tools. B.S. Mechanical Engineering Boston University, submitted to the Department of Mechanical Engineering in Partial Ful- 
filment of the Requirements for the Degree of Master of Science, Massachusetts Institute of Technology.

[6] MathWorks: About Stewart Platform, url: https://www. mathworks.com/help/physmod/sm/mech/ug/about-thestewart-platform.html? requestedDomain=www.mathworks.com. Downloaded: 2017.09.25.

[7] Gough V. E. (1956-1957), Contribution to discussion of papers on research in automobile stability, control and tyre performance. Proc. Auto Div. Inst. Mech. Eng., 392-394.

[8] Stewart D. (1965-1966), A platform with six degrees of freedom. Proc. Institution of Mechanical Engineers (UK). 180(Pt 1, No 15).

[9] Lazard D., Merlet J.-P. (1994), The (true) Stewart platform has 12 configurations. In: Proceedings of the 1994 IEEE International Conference on Robotics and Automation. p. 2160. ISBN 0-8186-5330-2. doi:10.1109/ROBOT.1994.350969
[10] Tompkins E. (1981), The History of the Pneumatic Tyre. Dunlop. pp. 86, 91. ISBN 0-903214-14-8

[11] Advanced Robotics and Mechanism Applications: Analysis and Synthesis of Parallel Robots for Medical Applications, A.R.M.A. Research Laboratory, Columbia University, Department of Mechanical Engineering, http://www. columbia.edu/cu/mece/arma/people/nabil_simaan/ms_research.shtml. Downloaded 2017.09.25.

[12] PI: Hexapod +3 Redundant Measuring Systems / Legs Medical Robot with Highest Levels of Reliability, http:// www.pi-usa.us/news/newsletter/old/27/hexa.html\#IPA. Downloaded: 2017.09.25.

[13] MICROMO: Hexapod Surgery Assistant: Servo Motor Applications, Miniature Hexapods Used In Spinal Surgery, url: https://www.micromo.com/applications/medical-lab-automation-equipment/application-case-study-micro-precise-surgery-assistant, Downloaded: 2017.09.25. 